\title{
Bell's palsy following COVID-19 vaccination with high CSF antibody response
}

\author{
Mark Obermann $^{1}$ (1) Maliqe Krasniqi ${ }^{1} \cdot$ Nadja Ewers $^{2} \cdot$ Jaber Fayad $^{1} \cdot$ Uta Haeberle $^{1}$
}

Received: 31 March 2021 / Accepted: 12 July 2021 / Published online: 29 July 2021

(c) Fondazione Società Italiana di Neurologia 2021

\section{Dear Sirs,}

The causal relationship between Bell's palsy and COVID19 mRNA vaccines is unclear. BioNTech/Pfizer included more than 43,000 people in their development program, out of which half received the active vaccine, while the others received placebo. Four cases of Bell's palsy were reported in the vaccine group, while none happened in the placebo group [1]. The Moderna trial program (mRNA-1273, Moderna) included 30,000 people and also reported 4 cases of Bell's palsy, with only three in the vaccine group and one in the placebo group [2]. The time interval between vaccination and onset of Bell's palsy in all study patients ranged widely and occurred at 3,9,37, and 48 days after vaccination. The vaccine security program of the Paul-Ehrlich-Institute (PEI), Germany reported 10 cases of Bell's palsy so far that developed between hours and 12 days after vaccination (report date 31.01.2021) [3]. One case was submitted anonymously with insufficient information, so that it is unclear whether this is an eleventh case.

\section{Case presentation}

A 21-year-old nurse working in a retirement home developed right facial palsy 2 days after receiving her first dose of SARS-CoV-2 mRNA vaccine Comirnaty (BNT162b2, BioNTech/Pfizer). She reported that she experienced a slight tingling sensation the day after vaccination and woke up the second day after vaccination with facial muscle paralysis (Fig. 1). There was minimal muscle tenderness at injection site but no other side effects. She presented to

Mark Obermann

mark.obermann@uni-due.de

1 Department of Neurology, Hospital Weser-Egge, Brenkhäuser Str. 71, 37671 Höxter, Germany

2 MVZ Diamedis GmbH, Bielefeld, Germany our emergency unit 10 days after symptom onset. Magnetic resonance imaging (MRI) of her head showed no intracranial lesion, or other pathology. Cerebrospinal fluid (CRF) analysis was unremarkable with 2 cells/ $\mu \mathrm{l}$, normal protein $(0.41 \mathrm{~g} / \mathrm{l})$, lactate, and glucose. Herpes simplex type 1 (HSV), varicella-zoster-virus (VZV), measles virus, and Borrelia antibodies were negative. Motor-evoked potentials (MEP) to the musculus nasalis showed normal latency and amplitude on the left side from cortical, temporal stimulation over the petrous bone, as well as stimulation over the stylomastoid foramen, while the right side did not elicit any potential (Fig. 2). SARS-CoV-2 antibodies against the S1-protein were detectable in blood and CSF using the commercially available Anti-SARS-CoV-2-ELISA (Euroimmun) demonstrating a good immune response to the first vaccination (SARS-CoV-2-IgA: 4.5 ratio, neg: $<0.8$, pos: $>1.0$; SARS-CoV-2-IgG: 5.4 ratio, neg: $<0.8$, pos: $\geq 1.0$, SARSCoV-2-IgG quant. $>288.0 \mathrm{BAU} / \mathrm{ml}$ (BAU $=$ binding antibody units)). SARS-CoV-2-IgG AI (antibody index) serum was $64.3 \mathrm{RU} / \mathrm{ml}(\mathrm{RU}=$ relative units of quantification), CSF was $19.8 \mathrm{RU} / \mathrm{ml}$, and SARS-CoV-2-IgG AI was 86 . Total IgG serum was $927 \mathrm{mg} / \mathrm{dl}$; total IgG CSF was $33 \mathrm{mg} / \mathrm{l}$. The coronavirus SARS-CoV-2-RNA test was negative to exclude acute infection (Roche Elecsys Anti-SARS-CoV-2 negative: 0.07 COI (cutoff index), neg: $<1.0$, pos: $\geq 1.0$ ).

The patient was treated with oral prednisolone $100 \mathrm{mg}$ per day over 5 days, was shown face muscle training, and received eye protecting ointment and an overnight eye patch. She was discharged 3 days after admission and reported that she tolerated treatment well and started to regain lid closure without substantial improvement of her lower facial muscles.

\section{Discussion and conclusions}

This would be the eleventh confirmed case in Germany since the start of the surveillance program accompanying the vaccination efforts (27.12.2020-31.01.2021) out 
Fig. 1 Facial nerve paralysis (A) and after grimacing (B)

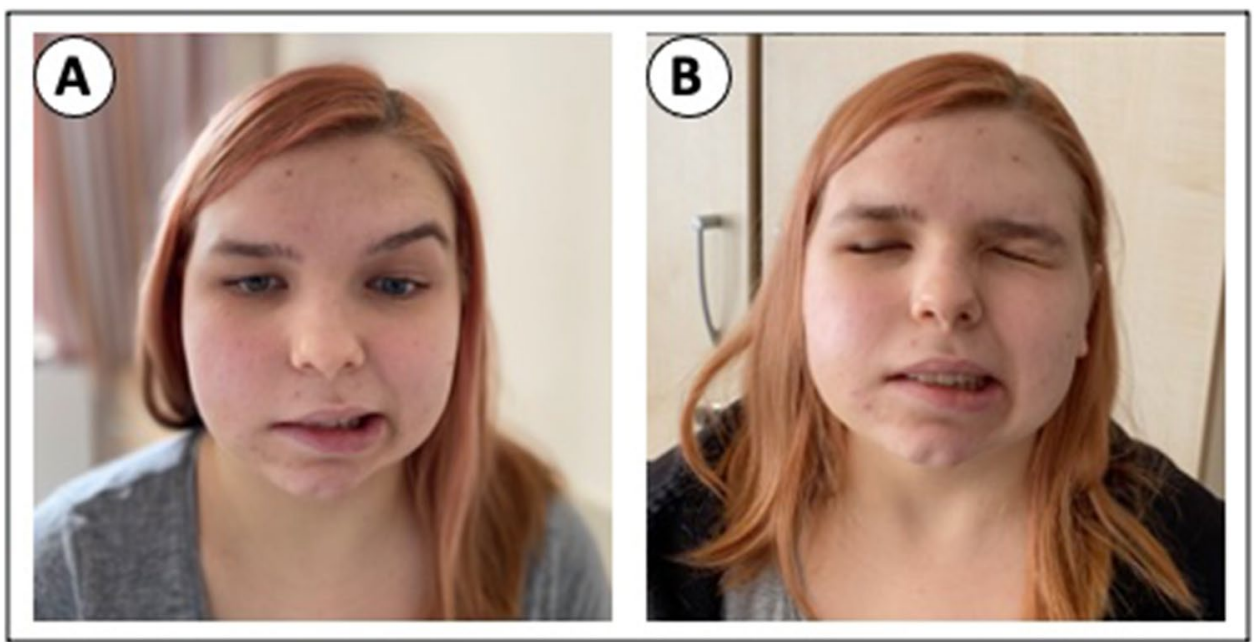

Fig. 2 Motor-evoked potentials showing good stimulation response from nasal muscle (A) and no response on the affected side with facial nerve paralysis (B)

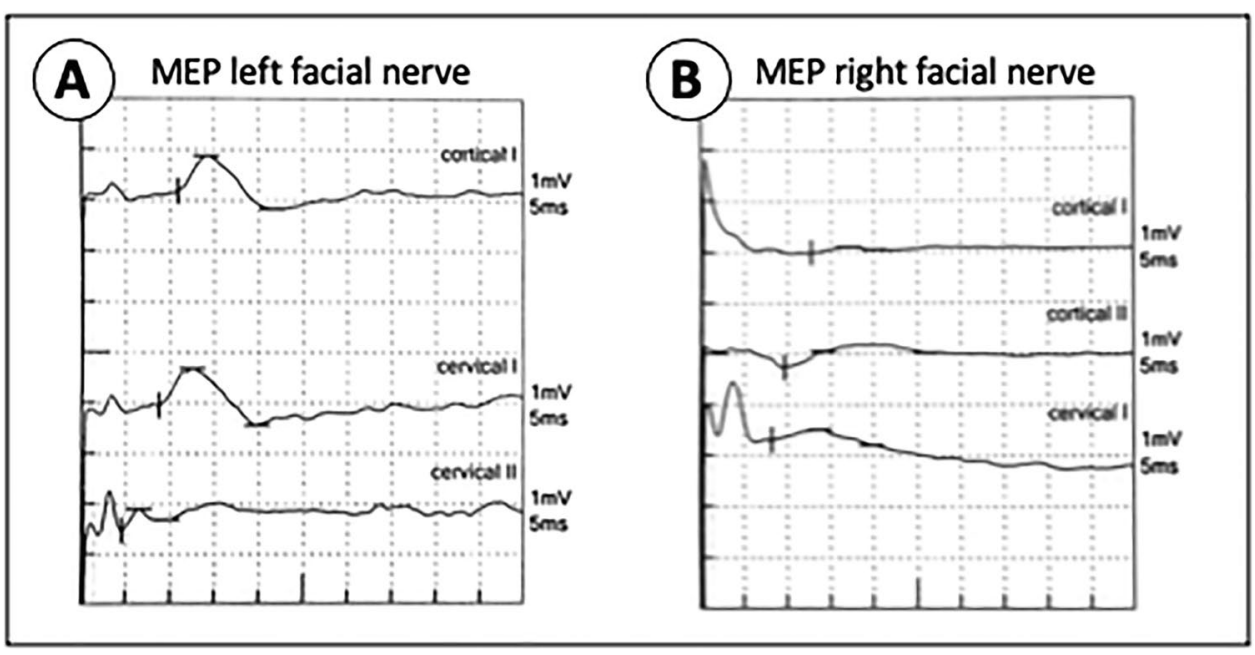

of 2,432,766 people that were vaccinated (2.2 per 100,000 persons) [3]. Even though the causal relationship between vaccination and onset of Bell's palsy remains uncertain, the temporal relationship of clinical symptoms and vaccination is much more plausible than what was reported from the clinical trials. In two reported cases, the onset of symptoms was only hours from vaccination, so that an effect from vaccination seems rather unlikely. In other cases, it remains uncertain whether the facial nerve was genuinely affected [1-3].

We tried to clearly demonstrate the affection of the facial nerve using motor-evoked potentials and also demonstrated the successful vaccination by quantification of antibodies against the spike protein (S1). The high antibody index (AI) comparing serum and CSF might even be interpreted as a potentially overwhelming vaccine response, or more than usual penetration in the central nervous system, so that the facial nerve could get affected. Would a high AI be a risk factor to develop Bell's palsy after vaccination and should it lead to early diagnosis and swift treatment in order to prevent long-term damage to the facial nerve?

Bell's palsy is usually a benign condition with high spontaneous recovery rates of $71 \%$. Sequelae were generally slight in $12 \%$ of patients, mild in $13 \%$, and severe only in $4 \%$ [4].

The estimated incidence rate of Bell's palsy in the general population ranges from 15 to 30 cases per 100,000 person-years. Since the 40,000 vaccine arm participants were followed for a median of 2 months, the combined safety population receiving vaccine across the two trials represents roughly 6700 person-years of observation time for an expected incidence of Bell's palsy of one to two cases, in line with the single observed case in the combined placebo arms. Therefore, the observed incidence of Bell's palsy in the vaccine arms is between 3.5 and 7 times higher than would be expected in the general population $[5,6]$.

A recent study form northern Italy demonstrated a higher incidence of Bell's palsy in patients suffering from 
COVID-19 than would be expected from data of the previous year in that same region, so that an immunologic mechanism might be associated with the infection as well as with the vaccination [7]. This hypothesis is emphasized by the fact that other viral vaccines, e.g., hepatitis B recombinant vaccine or influenza vaccine, were also suspected to lead to transient facial nerve paralysis $[8,9]$. Mimicry of host molecules or bystander activation of dormant autoreactive $\mathrm{T}$ cells was discussed as possible pathophysiological mechanism [10]. The vaccine might also induce innate immune activation and production of interferon proteins leading to facial nerve palsy [11]. More research is needed to unravel the potential pathophysiological mechanisms or to assure an incidental coexistence of vaccination and Bell's palsy, respectively.

The causal relationship between vaccination and the development of Bell's palsy remains uncertain. The temporal relationship to the vaccination should encourage health care professionals to be vigilant toward people developing Bell's palsy after SARS-CoV-2 vaccination with mRNA vaccine. Diagnosis and treatment should be swift and decisive.

Author contribution $\mathrm{MO}$ and $\mathrm{UH}$ wrote the first draft of the manuscript, MK and JF diagnosed and treated the patient, and NE did the blood and CSF analysis. All authors read and approved the final manuscript.

Data availability Not applicable.

\section{Declarations}

Ethical approval Ethics approval was waived.

Consent for publication Written informed consent for publication of their clinical details and/or clinical images was obtained from the patient. A copy of the consent form is available for review.

Conflict of interest The authors declare no competing interests.

\section{References}

1. Polack FP, Thomas SJ, Kitchin N et al (2020) Safety and efficacy of the BNT162b2 mRNA Covid-19 vaccine. N Engl J Med 383(27):2603-2615

2. Baden LR, El Sahly HM, Essink B et al (2021) Efficacy and safety of the mRNA-1273 SARS-CoV-2 vaccine. N Engl J Med 384(5):403-416

3. Paul-Ehrlich-Institute. sicherheitsbericht-27-12-bis-31-01-21.pdf [Internet]. [cited $2021 \mathrm{Feb} 6$ ]. Available from: https://www.pei. de/SharedDocs/Downloads/DE/newsroom/dossiers/sicherheitsberi chte/sicherheitsbericht-27-12-bis-31-01-21.pdf?_blob=publi cationFile \&v $=5$. Accessed 2 Feb 2021

4. Hughes GB (1990) Practical management of Bell's palsy. Otolaryngol-Head Neck Surg 102(6):658-663

5. Grogan PM, Gronseth GS (2001) Practice parameter: steroids, acyclovir, and surgery for Bell's palsy (an evidence-based review): report of the Quality Standards Subcommittee of the American Academy of Neurology. Neurology 56(7):830-836

6. Mustafa AHK, Suleiman AM. Bell's palsy: a prospective study. Int J Dent [Internet] 2020 [cited 2021 Jan 31];2020. Available from: https://www.ncbi.nlm.nih.gov/pmc/articles/PMC7102454/. Accessed 2 Feb 2021

7. Codeluppi L, Venturelli F, Rossi J et al (2021) Facial palsy during the COVID-19 pandemic. Brain Behav 11(1):e01939

8. Paul R, Stassen LFA (2014) Transient facial nerve paralysis (Bell's palsy) following administration of hepatitis B recombinant vaccine: a case report. Br Dent J 216(2):69-71

9. Mutsch M, Zhou W, Rhodes P et al (2004) Use of the inactivated intranasal influenza vaccine and the risk of Bell's palsy in Switzerland. N Engl J Med 350(9):896-903

10. Principi N, Esposito S (2020) Do vaccines have a role as a cause of autoimmune neurological syndromes? Front Public Health 8:361

11. Ozonoff A, Nanishi E, Levy O (2021) Bell's palsy and SARSCoV-2 vaccines. Lancet Infect Dis 21(4):450-452

Publisher's note Springer Nature remains neutral with regard to jurisdictional claims in published maps and institutional affiliations. 